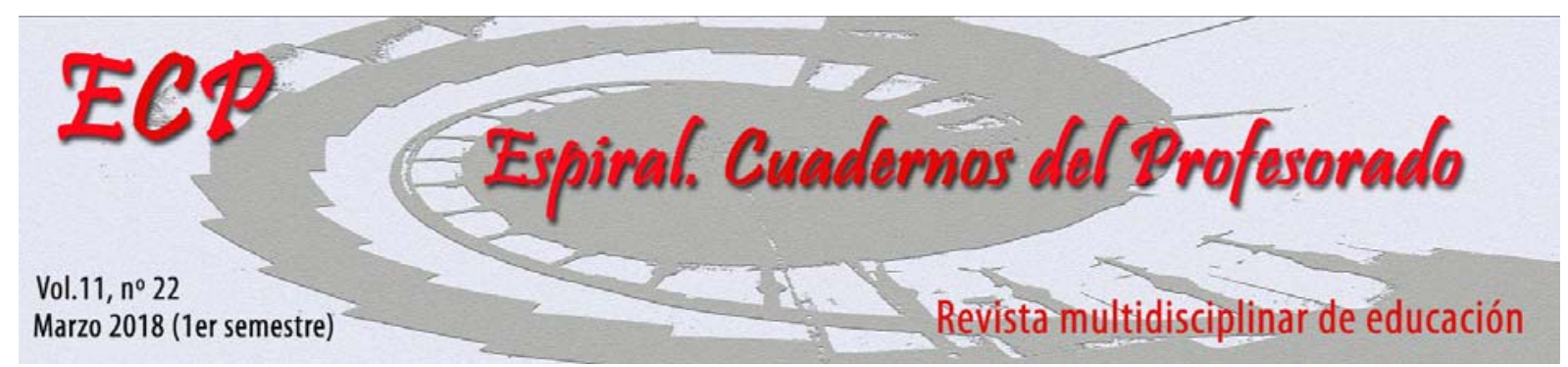

\title{
Cuestionario de evaluación de competencias personales y prosociales aplicado a las Actividades Físicas en el Medio Natural
}

\section{Questionnaire for the evaluation of personal and prosocial skills applied to physical activities in the natural environment}

\author{
Tomás Peire Fernández y Joan Estrada Aguilar
}

Universidad Autónoma de Barcelona, España

\begin{abstract}
RESUMEN: En este artículo se desarrolla una línea de trabajo basada en la investigación que estamos realizando en la Universitat Autònoma de Barcelona (UAB), para mejorar la adquisición de las competencias personales y prosociales en los grados de Educación Infantil y de Educación Primaria. En él se explica cómo hemos trasladado los resultados de dicha investigación, inicialmente planteados desde una perspectiva general de la formación de los maestros a través del prácticum, a la enseñanza de las actividades físicas en la naturaleza, vinculándolos a la evaluación de las competencias. La aportación innovadora más relevante ha sido crear una herramienta, en forma de cuestionario o rúbrica de evaluación formativa, que tiene la finalidad de facilitar la comprobación y la valoración de los logros que consigue el alumnado respecto a las competencias personales y prosociales. Argumentaremos que el cuestionario es una herramienta útil tanto para el profesorado que incluye en sus programas las actividades físicas en la naturaleza, como para el alumnado que las realiza.
\end{abstract}

Palabras clave: competencias pro-sociales, competencias personales, actividad física en la naturaleza, cuestionario de evaluación.

\begin{abstract}
This article develops a line of work based on research that we are doing at the Universitat Autònoma de Barcelona (UAB), to improve the acquisition of personal and proactive skills in the levels of Early Childhood Education and Primary Education. We explain how we have translated the results of this research, initially raised from a general perspective of the training of teachers through practical, to the teaching of physical activities in nature, linking them to the assessment of skills. The most relevant innovative contribution is a tool, in the form of a questionnaire of formative evaluation. That has the purpose of facilitating the verification and the evaluation of the students' achievements with respect to those competences. As will be shown below, it is a useful tool for teachers who want to include activities in natural environments as well as for the students who carry them out.
\end{abstract}

Key words: proactive competences, personal competences, physical activity in nature, evaluation questionnaire.

Peire Fernández, T. y Estrada Aguilar, J. (2018). Cuestionario de evaluación de competencias personales y prosociales aplicado a las Actividades Físicas en el Medio Natural. Espiral. Cuadernos del Profesorado, 11(22), 79-86

Fecha de recepción: 12/05/2017

Fecha de aceptación: 09/11/2017

Correspondencia: tomas.peire@uab.cat

(T. Peire Fernández)

\section{Fundamentos de la propuesta de cuestionario competencial}

La propuesta de cuestionario de evaluación se basa en dos líneas de trabajo y de experiencias, que se complementan. Por una parte, la experiencia adquirida en la organización y la docencia de Actividades Físicas en el Medio Natural (AFIMENA) en la UAB, con alumnado de magisterio, desde el año 1992 Peire, Estrada, Magallón (2011). Por otra parte, los trabajos realizados por un grupo de 
investigación del que formamos parte los autores de este artículo, denominado: Sumando Compromiso Educativo, que trabaja en el proyecto Implementación y evaluación de un prácticum con mayor compromiso social: creación de escenarios formativos colaborativos para el desarrollo social y profesional de los futuros maestros, adscrito a un amplio programa para la Mejora de la Formación Inicial de los grados de Educación Infantil y de Educación Primaria (MIF), de la Generalitat de Catalunya, que se acompaña con un plan de financiación denominado MIF-ARMIF.

Este grupo plantea como objetivo final de su investigación, provocar cambios en el modelo formativo de los maestros, creando estrategias de formación competencial que permitan una participación más activa y substancial entre escuela y universidad, entendiendo la educación como un espacio compartido entre diferentes agentes sociales. Deseamos una escuela más creativa y democrática, que construya mecanismos y herramientas desde las necesidades reales del día a día, y con una implicación de maestros y maestras más holística, más comprometida, haciendo énfasis en sus competencias personales y sociales.

Una de las herramientas formativas que el grupo ARMIF ha desarrollado es el Cuestionario de evaluación competencial. Aunque se ha diseñado para su utilización en el desarrollo de los prácticums de los grados de Educación Infantil y de Educación Primaria, está concebido como recurso abierto y dúctil, para que se pueda adaptar a otras finalidades, áreas de conocimiento, entornos formativos, etc.

Justamente eso es lo que hemos hecho y vamos a explicar: adaptar una herramienta genérica de evaluación a un uso específico. El modelo se ha adaptado para ser aplicado en la formación de profesorado de Educación Primaria de la mención en Educación Física (MEF) de la UAB, para la evaluación de las competencias personales y prosociales programadas a través de las Actividades Físicas en el Medio Natural. Servirá de ejemplo para demostrar la versatilidad del instrumento. Partimos de la base de que las competencias personales y prosociales son imprescindibles para el ejercicio profesional de los maestros y maestras. Además, por supuesto, de las competencias profesionales más específicas. En los siguientes apartados intentaremos profundizar en este argumento.

\section{La relación con la naturaleza favorece la adquisición de competencias.}

El cuestionario de evaluación que proponemos se ajusta al modelo actual de programación curricular que orienta y condensa la formación alrededor del concepto de competencia. Según el modelo, los objetivos, los contenidos formativos, las metodologías y los recursos que se aplican en los procesos de formación profesional convergen en el logro de competencias.

Por otra parte, la propuesta se sitúa en el ámbito de las actividades de enseñanza-aprendizaje en el medio natural. La naturaleza ofrece condiciones ideales para desarrollar las competencias personales y prosociales Estrada, Peire, y Magallón (2011): "Muchos aspectos necesarios para el crecimiento personal y el progreso de la autonomía y de los comportamientos responsables, se pueden fomentar de forma muy eficaz a través de la realización de las Actividades Físicas en Ambiente Natural".

Se viven situaciones singulares en un entorno estimulante y atractivo, también propicias para el desarrollo personal y social. Los valores formativos implícitos en las actividades físicas en la naturaleza, entre otros, son los siguientes:

- la necesidad y el estímulo de observar, comprender y adaptarse a la naturaleza; imprevisible, diversa, atractiva, ...

- la imprescindible consideración de las necesidades de los demás en relación a las propias, en un entorno no habitual,

- el aprendizaje de conceptos, de técnicas y de uso de materiales contextualizados,

- la observación de normas de seguridad específicas,

- el uso del vocabulario y de las expresiones propias del medio,

- la renuncia a la comodidad, la rutina y los recursos y usos de la vida cotidiana, 
- la superación de exigencias no habituales y de constantes retos,

- la identificación de las limitaciones propias y de los demás en entornos desconocidos,

- el sentido y el valor de la sostenibilidad, el reconocimiento de la fuerza de la naturaleza, pero también de su fragilidad, y de la necesidad de respetarla.

Pero atención, la potencialidad formativa de las AFIMENA, no resultará eficaz si no van acompañadas de intencionalidad y acción educativa, y son guiadas con las orientaciones, criterios, métodos y técnicas adecuadas, y el buen criterio.

Aunque nos centraremos en las competencias personales y prosociales y no profundizaremos en esta ocasión en las competencias profesionales específicas, que el alumnado de magisterio de educación física debe desarrollar para las AFIMENA, queremos destacar tres competencias profesionales como ejemplo de las que incluiríamos en un cuestionario más enfocado a ellas. Elegimos tres que consideramos demostrativas. Dos de carácter transversal e interdisciplinar, referidas a la sostenibilidad y a la inclusividad, y una referida a la organización.

La competencia derivada de la sostenibilidad, podemos llamarla Competencia para la valoración, el respeto y la defensa del medio natural, incluye la sensibilización hacia el medio ambiente, la identificación de los riesgos que amenazan y el conocimiento de las formas de preservación medioambientales. La educación para la sostenibilidad ha de ser una prioridad en la intervención educativa, desde la perspectiva del compromiso social.

La competencia sobre la educación inclusiva, a la que podemos llamar Competencia para el respeto y la inclusión de las personas con necesidades educativas especiales. Es una obligación moral y profesional posibilitar la práctica de las AFIMENA a toda persona que lo desee.

El interés creciente de programas inclusivos de estas actividades por parte de la ciudadanía, que ha activado la formación de profesionales para esta finalidad y la aparición y el uso de recursos adaptados como la "Jöelette", la barra direccional, las sillas para esquí, el acompañamiento de guías, etc., son claros exponentes de los esfuerzos que se aplican cada vez de forma más decidida.

La competencia sobre organización podría enunciarse como Competencia para la conducción y la gestión de grupos en el medio natural. La complejidad de organizar con seguridad y garantías las AFIMENA para evitar riesgos y cumplir con el programa y los objetivos planteados en cada caso, se traduce en una competencia esencial que han de desarrollar los profesionales responsables.

Esta competencia incluye factores relativos a: la capacidad de prever necesidades, de informar eficazmente a los participantes, al conocimiento del medio y de técnicas de orientación, al conocimiento de primeros auxilios y de medidas de seguridad, al conocimiento de herramientas y recursos específicos para las diferentes AFIMENA, etc.

Defendemos el paradigma de la Educación Física que pone en relación al hombre con la naturaleza bajo el principio de la sostenibilidad y el compromiso del respeto a las personas y al medio. Las actividades físicas en el medio natural hay que verlas como recurso imprescindible para potenciar el conocimiento de uno mismo, la autonomía personal y la relación de calidad con los demás y con el entorno natural.

Nos centramos en explorar y explotar las posibilidades de la formación en competencias que posibilitan las AFIMENA. Para ello proponemos la utilización de un instrumento de evaluación, en formato de cuestionario, que facilitará la tarea al profesorado, y el progreso del alumnado.

El cuestionario es sencillo de aplicar y aporta una doble finalidad: explica algunas de las competencias prosociales y personales más relevantes, para hacer reflexionar a alumnado y profesorado. También ayuda a valorar si se producen progresos.

Este cuestionario aconsejamos sea aplicado más de una vez, una antes de empezar el programa de actividades y otra al final, esta última para revisar y reflexionar sobre las actitudes y comportamientos producidos en el proceso, interiorizar la experiencia personal y valorar los cambios experimentados. 
La utilización de instrumentos de evaluación sobre competencias personales y prosociales en el medio natural imprime una consideración más elevada a su importancia formativa.

\section{Procedimiento de construcción y validación del cuestionario}

La construcción del cuestionario se desarrolló a través de cuatro estrategias y en tres fases.

\section{Estrategias:}

- Grupo de investigación. Análisis de documentos, debates.

- Grupos focales con maestros y maestras de escuelas de prácticas. Análisis de resultados.

- Grupo de Investigación. Redactado final del cuestionario general.

- Autores del artículo. Adaptación a un uso específico: AFIMENA. Redactado final.

\section{Primera fase}

En una primera fase se realizaron estudios y debates con los miembros del grupo ARMIF compuesto por profesorado de la UAB y maestras y maestros de EI y de EP de escuelas de prácticas. Este trabajo consistió en investigar y decidir sobre las competencias que considerábamos más importantes y significativas.

Se valoró la selección de competencias a partir de la revisión de la literatura científica en la materia y de las competencias que se especifican en la guía docente de las asignaturas de los grados que se imparten en la Facultad.

La principal dificultad que surgió fue la concreción de cada una de ellas. Resulta complejo definir algunas de las competencias ya que están interrelacionadas y se complementan con otras, es decir, el desarrollo de una competencia implica, a menudo, también a otras.

El primer diseño de cuestionario, el más completo y global, incluye 32 competencias claramente diferenciadas y definidas conceptualmente. No se da por cerrado, puesto que en el futuro pueden identificarse otras competencias. Son las siguientes:

$\begin{array}{lll}\text { Adaptación } & \text { Diálogo } & \text { Pensamiento crítico } \\ \text { Argumentación } & \text { Empatía } & \text { Resiliencia } \\ \text { Asertividad } & \text { Entusiasmo/pasión } & \text { Respeto } \\ \text { Autonomía } & \text { Escucha activa } & \text { Responsabilidad } \\ \text { Autoconfianza } & \text { Esfuerzo } & \text { Seducción/persuasión } \\ \text { Autocontrol } & \text { Expresión } & \text { Superación } \\ \text { Autocrítica } & \text { Interdisciplinariedad } & \text { Trabajo en equipo } \\ \text { Compromiso } & \text { Liderazgo } & \\ \text { Comunicación } & \text { Mediación } & \\ \text { Concentración } & \text { Prudencia/previsión } & \\ \text { Control emocional } & \text { Objetividad Organización } & \\ \text { Cooperación } & & \\ \text { Creatividad/imaginación } & & \end{array}$

\section{Segunda fase}

En la segunda fase, el cuestionario fue expuesto y tratado en otros 3 ámbitos para su adecuación y validación:

A. El cuestionario se mostró a maestras y maestros de escuelas que reciben estudiantes de la $\mathrm{UAB}$ en prácticas. Recogimos opiniones sobre el primer cuestionario de 32 competencias, sobre el acierto de su conceptualización, sobre su importancia, o sobre las dificultades para evaluarlas, entre otros aspectos. 
B. Se organizaron grupos focales de maestras y maestros, moderados por profesorado de la $\mathrm{UAB}, \mathrm{y}$ en diversos centros de educación infantil y primaria se realizaron debates en torno a las competencias, para seleccionar las prioritarias. Los centros participantes fueron los siguientes: Encants, Polinyà, La Sínia, El Martinet, Cavall Fort, Tricicle, Marina, Escola Bellaterra, Carles Buïgas y Rivo Rubeo.

C. Se procedió al redactado final de la definición de cada competencia con revisión por parte del grupo de investigación. Finalmente se redactó en catalán y en castellano, con las correspondientes revisiones lingüísticas.

Se ajustó el cuestionario para que ofreciera las características siguientes:

$\checkmark$ Versátil. Abierto a la inclusión de las competencias que se consideren adecuadas en cada situación y para las finalidades convenientes. Las competencias a evaluar pueden variar, como también el número de ellas.

$\checkmark$ Adaptable. Al contexto educativo en que se desee aplicar, a la tipología del alumnado, a la duración del programa formativo, etc.

$\checkmark$ Didáctico. Formativo en el sentido de ofrecer información competencial destacada, expresada de forma comprensible y contextualizada.

Como resultado y con la participación de los autores se publicó un cuestionario de competencias personales. Equip de Recerca $\Sigma$ Compromís Educatiu (2016).

\section{Tercera fase}

La tercera fase sirvió para adaptar el cuestionario a un programa de actividades físicas en el medio natural en los estudios de magisterio de Educación Física. Del diseño general de cuestionario previsto para ser aplicado en el prácticum de magisterio, pasamos a su ajuste para ser aplicado en un entorno formativo más específico. Concretamente en la mención de Educación Física, con estudiantes que realizan un programa de AFIMENA en diferentes momentos de su formación.

Se consultó a profesionales de la educación en general y a especialistas de educación física y de los deportes en el medio natural, que aportaron opiniones muy interesantes sobre el objeto de estudio. El proceso de debate y de reflexión para la edición del cuestionario específico, se orientó a seleccionar aquellas competencias personales y prosociales que se manifiestan en la práctica de actividades físicas en el medio natural, y que son más fácilmente identificables y evaluables, tanto por el profesorado como, y más determinante, por parte del alumnado participante.

\section{Funciones del cuestionario de evaluación de competencias personales y prosociales}

La aplicación del cuestionario de evaluación puede servir para diversas funciones, no excluyentes:

1. para que los docentes evalúen la evolución de sus estudiantes

2. para la autoevaluación del alumnado participante

3. para la evaluación entre iguales o coevaluación, como herramienta de dialogo y reflexión.

4. para la evaluación de la eficacia del programa

En todo caso, debería servir para colaborar para el logro de los objetivos formativos, como elemento influyente de desarrollo competencial y para el crecimiento personal de su alumnado.

Presentamos el cuestionario de competencias con escala de valoración. El valor $\mathbf{0}$ expresa la nula adquisición de la competencia. El valor 7, el máximo nivel. El nombre de la competencia va acompañado de una definición breve pero suficiente, con referencia al medio natural que ayuda a la comprensión de los conceptos que incluye (ver Anexo 1). 


\section{Conclusiones}

El cuestionario de valoración que hemos presentado afronta la difícil tarea de evaluar competencias. Hemos pasado de considerar que las actividades físicas en el medio natural pueden ser propicias para el desarrollo integral de la persona y específicamente para la adquisición de competencias personales y prosociales, a intentar comprobarlo. Para ello hemos trabajado en la siguiente dirección:

1. Definir algunas competencias personales y prosociales implicadas en las AFIMENA como guía para su desarrollo e implementación en los programas formativos.

2. Valorar la evolución de las competencias personales y prosociales entre el alumnado participante en los programas de AFIMENA a través de instrumentos de evaluación formativa, como el Cuestionario de Valoración de Competencias Personales y Prosociales en el Medio Natural.

En la UAB hemos empezado a aplicarlo, pero no podemos ofrecer todavía resultados significativos y fiables.

El tratamiento de los resultados de los cuestionarios puede favorecer, en el futuro, la evolución y consolidación de programas de AFIMENA más eficaces y fundamentados, y el inicio de interesantes líneas de investigación sobre esta temática. Por otra parte, conviene recordar el formato abierto y libre del cuestionario para ser adaptado a cada circunstancia y objetivo.

Uno de los usos adaptados, podría ser el ajuste de su contenido como herramienta de formación y de evaluación orientada concretamente hacia la definición de las competencias profesionales específicas que los maestros y maestras han de adquirir para poder responsabilizarse, con conocimiento y seguridad, de las AFIMENA en sus programaciones de Educación Física escolar.

\section{Referencias}

Comes, P., Peire, T., Morón, M., y Guitart, J. M. (2013). ApS y la cultura de la responsabilidad profesional. Proyectos de ApS en el prácticum de la formación inicial de maestros y maestras de las especialidades de Educación Infantil y Educación primaria en la UAB. Barcelona: ICE UB. Colección Educación y Comunidad.

Elias, M., Tobias, S., y Frieldlander, B. (2001): Educar con inteligencia emocional. Barcelona: Plaza \& Janés.

Estrada, J., Peire, T., y Magallón, J. (2011). Potencial educativo de las actividades físicas en el ambiente natural. Actividades Físicas em Ambiente Natural. As actividades na neve (coordinador: Petrica, J.M.). Castelo Branco, Portugal: Ediciones IPCB.

Equip de Recerca $\Sigma$ Compromís Educatiu (2016). Qüestionari d'avaluació del desenvolupament de les competències personals i prosocials. Recuperado de https://ddd.uab.cat/record/145653. Bellaterra: Publicacions UAB.

Gervais, C. y Desrossiers, P. (2005). L'école lieu de formation d'enseignants. Questions et repères pour la formation de stagiaires. Québec: Les Presses de l'Université de Laval.

Guillen, R., Lapetra, S., y Casterad, J. (2010). Actividades en la naturaleza. Barcelona: INDE.

Iglesias Cueto, J. A. (2005). Actividades en la Naturaleza. Fichero. Barcelona: INDE.

Larrauri, M. (2012). La educación según John Dewey. Valencia: Tandem.

Trowler, V. (2010). Student engagement review. N. York: The Higher Education Academy.

Marina, J.A. y Bernabeu, R. (2007). Competencia social y ciudadana. Barcelona: Graó.

UNESCO (2009). Conferencia Mundial sobre la Ed. Superior 2009: La nueva dinámica de la educació superior y la investigación para el cambio social y el desarrollo. París: Ref. ED. 2009/CONF.402/2. Recuperado de http://www.unesco.org/education/WCHE2009/comunicado_es.pdf. 


\section{Anexo 1 \\ CUESTIONARIO DE VALORACIÓN DE COMPETENCIAS PERSONALES Y PROSOCIALES EN EL MEDIO NATURAL}

Nombre y apellidos: Puede responder el alumno/a autoevaluándose, y el/la docente, evaluando.

Puntúa de 0 a 7 el grado de adquisición de las siguientes competencias personales y prosociales

\begin{tabular}{|c|c|c|}
\hline $\begin{array}{l}\text { Competencias personales } \\
\text { y prosociales }\end{array}$ & $\begin{array}{l}\text { Valor de } \\
\text { 0 a } 7\end{array}$ & $\begin{array}{l}\text { Definición de las competencias contextualizadas } \\
\text { en el medio natural }\end{array}$ \\
\hline $\begin{array}{l}\text { 1.- Creatividad e } \\
\text { imaginación }\end{array}$ & & $\begin{array}{l}\text { Ser capaz de generar ideas, respuestas y propuestas innovadoras y } \\
\text { alternativas } \\
\text { Conocer nuevos espacios naturales puede proporcionar inagotables } \\
\text { posibilidades para interactuar a través de actitudes y metodologías } \\
\text { dinámicas y proactivas. El descubrimiento de nuevos parajes es muy } \\
\text { estimulante y la evolución de las modalidades deportivas enriquece y } \\
\text { favorece la diversidad de experiencias. }\end{array}$ \\
\hline 2.- Concentración & & $\begin{array}{l}\text { Saber mantener la atención el tiempo necesario para realizar una } \\
\text { tarea } \\
\text { El medio natural exige nuevos aprendizajes y continuas adaptaciones } \\
\text { que conllevan la necesidad de observación y concentración. La } \\
\text { concentración en las AFIMENAS tiene mucho que ver con la seguridad } \\
\text { y el progreso en el dominio de conceptos y técnicas específicas. }\end{array}$ \\
\hline $\begin{array}{l}\text { 3.- Interdisciplinariedad } \\
\text { (transversal y global) }\end{array}$ & & $\begin{array}{l}\text { Saber llevar a cabo procesos relacionando contenidos de diferentes } \\
\text { áreas, con visión amplia e interactiva. } \\
\text { Permite mejorar la significatividad de los aprendizajes, a veces } \\
\text { complejos, así como sus interrelaciones y la coherencia entre ellos. } \\
\text { Como por ejemplo: acampada, geología, fauna, flora, manifestaciones } \\
\text { ambientales, del clima, el relato, la descripción, el dibujo, la pintura, } \\
\text { etc. }\end{array}$ \\
\hline 4.- Autoconfianza & & $\begin{array}{l}\text { Tener seguridad en las propias capacidades para resolver retos. } \\
\text { Descubrir las propias capacidades puede favorecer la mejora de la } \\
\text { seguridad en uno mismo. Por ejemplo: conseguir nuevos aprendizajes, } \\
\text { la superación de retos difíciles e inusuales, el acceso a lo desconocido, } \\
\text { ayudar a los demás,... }\end{array}$ \\
\hline 5.- Resiliencia & & $\begin{array}{l}\text { Saber gestionar la adversidad y las dificultades, con capacidad de } \\
\text { resistir. } \\
\text { Afrontar nuevas situaciones en entornos que no se conocen ni dominan } \\
\text { como los habituales, con las incomodidades, el cansancio y las } \\
\text { dificultades inherentes a las AFIMENA, proporciona oportunidades } \\
\text { óptimas para desarrollar esta competencia. }\end{array}$ \\
\hline 6.- Control emocional & & $\begin{array}{l}\text { Reconocer y regular las propias emociones para ajustarlas } \\
\text { adecuadamente a las necesidades del momento y del contexto. } \\
\text { Las propias circunstancias de adaptación al medio natural y a formas de } \\
\text { relación diferentes de las habituales pueden presentar numerosas } \\
\text { situaciones que exijan gestión y modulación emocional. }\end{array}$ \\
\hline
\end{tabular}




\begin{tabular}{|c|c|}
\hline 7.- Esfuerzo & $\begin{array}{l}\text { Insistir en la superación de dificultades para conseguir los logros } \\
\text { pretendidos. } \\
\text { En las AFIMENA los retos son constantes. Conllevan la realización de } \\
\text { continuos esfuerzos, a veces extraordinarios. Esta competencia abarca } \\
\text { las vertientes física, psicológica, cognitiva y sociológica. }\end{array}$ \\
\hline 8.- Asertividad & $\begin{array}{l}\text { Saber expresar los sentimientos, las emociones o los pensamientos } \\
\text { propios de manera libre y con seguridad, sin negar los derechos de } \\
\text { los demás y sin que los demás se sientan agredidos o manipulados. } \\
\text { Las AFIMENA pueden ser un marco ideal para establecer nuevas } \\
\text { relaciones sociales o fortalecerlas. Pactar, improvisar, asumir, escuchar } \\
\text { e interpretar, expresar opiniones personales y defenderlas, ... son } \\
\text { exigencias habituales en ese contexto. }\end{array}$ \\
\hline 9.- Adaptabilidad & $\begin{array}{l}\text { Saber adecuarse a cualquier contexto, relación o situación de forma } \\
\text { positiva. Condición que permite aceptar y acomodarse a los cambios. } \\
\text { Las AFIMENA se caracterizan por la diversidad de experiencias i por } \\
\text { lo imprevisto. Las situaciones cambian constantemente: la } \\
\text { organización, las relaciones personales, el clima, las condiciones de } \\
\text { vida, la alimentación, las comodidades, el trabajo en equipo, los } \\
\text { materiales y los recursos específicos, ... obligan a la adaptación } \\
\text { constante. }\end{array}$ \\
\hline 10.- Empatía & $\begin{array}{l}\text { Saber percibir y comprender el comportamiento, las emociones y los } \\
\text { sentimientos de otra persona o un grupo de personas. } \\
\text { El marco de relación social que ofrecen las AFIMENA presenta } \\
\text { muchas ocasiones para la interacción y la convivencia. Son } \\
\text { oportunidades para desarrollar la capacidad de comprender y de } \\
\text { hacerse entender. La necesidad de expresión, de observación y de } \\
\text { escucha son constantes. }\end{array}$ \\
\hline 11.- Cooperación & $\begin{array}{l}\text { Saber convivir y colaborar para conseguir objetivos compartidos. } \\
\text { En las AFIMENA la responsabilidad de las tareas acostumbran a ser } \\
\text { compartidas. Los desplazamientos en grupo, las actividades, el orden y } \\
\text { el transporte de los materiales, la preparación de la comida y el } \\
\text { compartirla, etc., son situaciones ideales para la cooperación y la } \\
\text { solidaridad. }\end{array}$ \\
\hline 12.- Respeto & $\begin{array}{l}\text { Consideración hacia los demás atendiendo a sus características y } \\
\text { situaciones personales y sociales. } \\
\text { El binomio de grupos heterogéneos y naturaleza origina que la } \\
\text { aceptación de las personas y del medio ambiente sea la actitud más } \\
\text { razonable y obvia. Sin esta actitud las AFIMENA no funcionan } \\
\text { correctamente. }\end{array}$ \\
\hline 13.- Autocrítica & $\begin{array}{l}\text { Mostrar disposición a recibir sugerencias, ideas, valoraciones y } \\
\text { críticas. Ser capaz de autoanalizarse y reconocer los propios errores y } \\
\text { limitaciones. } \\
\text { En contextos naturales, a menudo con menor conocimiento y } \\
\text { seguridad, aparecen un sinfín de oportunidades para la autoevaluación, } \\
\text { la revisión de las propias conductas, la crítica y la mejora personal. }\end{array}$ \\
\hline
\end{tabular}

